\title{
Aider les apprenantes et les apprenants à prendre de meilleures notes
}

\author{
Auteur(s) ${ }^{1}$ \\ Audrey Pépin, Université du Québec à Chicoutimi, Canada, \\ audrey.pepin1@uqac.ca \\ Patrick Giroux, Université du Québec à Chicoutimi, Canada, \\ patrickgiroux@uqac.ca \\ Odette Gagnon, Université du Québec à Chicoutimi, Canada, \\ odette gagnon@uqac.ca
}

\footnotetext{
${ }^{1}$ L'auteure principale a bénéficié des bourses du Conseil de recherche en sciences humaines du Canada (CRSH) et du Fonds de recherche du Québec - Société et culture (FRQSC) pour ses travaux de recherche à la maîtrise qui ont mené à la rédaction du présent article. Les autres auteurs ont, quant à eux, bénéficié d'une subvention de la Fondation de l'Université du Québec à Chicoutimi (FUQAC) pour un projet connexe sur la prise de notes.
} 


\section{REVUE HYBRIDE DE L'ÉDUCATION}

\section{Résumé}

La prise de notes (PDN) est une compétence complexe et importante, notamment par son un impact sur la mémorisation. Elle devrait être déployée tôt dans le parcours scolaire des apprenantes et des apprenants pour être maîtrisée. Étant donné que ce n'est pas le cas, les apprenantes et les apprenants vivent bien des difficultés lors de leurs PDN. L'article met en lumière les actions que les enseignantes et les enseignants peuvent poser pour faciliter la PDN afin de permettre aux apprenantes et aux apprenants de tirer des bienfaits de cette activité et ouvre sur une perspective didactique de la PDN.

Mots-clés : prise de notes; ressources attentionnelles; surcharge cognitive; interventions pédagogiques; didactique 


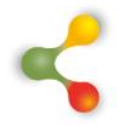

\section{REVUE HYBRIDE DE L'ÉDUCATION}

\section{Mise en contexte}

La prise de notes (PDN) en contexte d'écoute est une compétence importante qui devrait être déployée dès le secondaire, et peut-être même dès le primaire, pour être mieux maîtrisée par les élèves à leur entrée au collège ou à l'université (Romainville et Noël, 2003), où le contenu des cours est plus substantiel et le rythme de l'enseignement plus rapide (Pollet, 2001). La mauvaise maîtrise de cette dernière est souvent identifiée comme un obstacle rendant la transition vers le milieu universitaire difficile (Paivandi, 2015). Cet article vise à mettre en lumière les enjeux de la PDN de la perspective des apprenantes et des apprenants, mais aussi de celle des praticiennes et des praticiens qui peuvent adopter certaines pratiques afin de favoriser la compétence de PDN.

\section{Déroulement}

\section{Quels sont les bienfaits de la PDN ?}

On associe souvent la PDN aux études universitaires en raison du contenu plus substantiel des cours qu'il faut « enregistrer » quelque part pour pouvoir le réutiliser. Toutefois, la PDN peut s'avérer bénéfique à tous les apprenantes et les apprenants, peu importe leur niveau scolaire. En effet, l'activité de PDN rend les personnes actives lors de leur écoute et leur permet de mémoriser les informations en se les appropriant. Selon une étude menée par Einstein, Morris et Smith (1985) citée dans Boyle et Forchelli (2014), les personnes se souviendraient de $40 \%$ des informations qu'elles ont notées comparativement à $7 \%$ pour les informations qu'elles n'ont pas notées. De plus, la PDN permet de développer une mémoire externe fiable pour une appropriation ultérieure des informations (Piolat et Boch, 2004). Elle permet aussi de soulager la mémoire de travail lors de la résolution de problèmes complexes, comme la rédaction d'un texte ou la résolution d'un problème mathématique (Cary et Carlson, 2001) et de planifier les activités à venir (matière à réviser, examen, etc.) (Hartley, 2002).

\section{Pourquoi les apprenantes et les apprenants ont-ils tant de difficulté à maîtriser cette compétence ?}

La PDN est une compétence complexe, c'est-à-dire qu'elle nécessite que la personne prenant des notes réalise simultanément plusieurs opérations (Makany, Kemp, et Dror, 2009; Piolat, 2010) : comprendre les informations entendues, sélectionner les informations pertinentes et transcrire ces informations tout en les hiérarchisant (Piolat et Boch, 2004) et en marquant les liens qu'elles ont entre elles (Peverly et Sumowski, 2012). En plus de la gestion simultanée de toutes ces opérations, les apprenantes et les apprenants doivent prendre leurs notes dans l'urgence. 


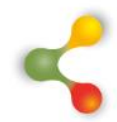

\section{REVUE HYBRIDE DE L'ÉDUCATION}

En effet, l'apprenante ou l'apprenant, dont le rythme d'écriture est plus lent que le rythme de parole de l'enseignante ou de l'enseignant, doit noter le plus rapidement possible les informations tout en réalisant les différentes opérations énoncées précédemment. De plus, l'apprenante ou l'apprenant doit tenir compte du renouvellement continu du message, c'est-à-dire mémoriser ce que le professeur dit dans sa mémoire à court terme, laquelle est limitée, tout en notant.

En somme, étant donné la gestion simultanée des opérations de compréhension et de production écrite, et l'urgence de la PDN, cette compétence entraine souvent des surcharges cognitives (Piolat, 2010) et est peu maîtrisée (Roussey et Piolat, 2003).

\section{Pour quelles stratégies les apprenantes et les apprenants optent-ils afin d'éviter de vivre des surcharges cognitives?}

Pour tenter d'accélérer le rythme de leur écriture et ainsi, tenter d'échapper à la surcharge cognitive causée par l'urgence, les apprenantes et les apprenants peuvent opter pour des procédés de resserrement, comme les abréviations et les symboles (Branca-Rosoff, 1998; Piolat, 2010). Plus ces derniers sont automatisés, plus leur usage est efficace (Piolat, 2004).

Une autre stratégie peut permettre d'échapper à la surcharge cognitive. II s'agit d'user stratégiquement de ses ressources attentionnelles en privilégiant soit la compréhension, soit la transcription (Piolat, 2004). Dans bien des cas, c'est la transcription qui est priorisée par les apprenantes et les apprenants. En notant le plus fidèlement possible ce que l'enseignante ou l'enseignant dit, l'apprenante ou l'apprenant peut restituer le tout fidèlement en vue d'un examen (Boch, 1998). Cette stratégie est à la fois avantageuse et désavantageuse. Il est vrai que de transcrire le message le plus fidèlement possible en vue de reporter sa compréhension peut paraitre une bonne méthode, surtout quand on pense au fait que la PDN n'a de sens que si les notes sont utilisées pour un travail ultérieur, notamment pour la révision (Boch, 2000). Toutefois, le fait d'écrire sans comprendre ne permet pas à l'apprenante ou à l'apprenant de prendre des notes efficaces, c'est-à-dire des notes dans lesquelles les informations pertinentes sont organisées, hiérarchisées et liées entre elles (Williams et Eggert, 2002). De plus, les informations ne sont traitées que superficiellement et ne sont donc pas encodées dans la mémoire à long terme (Aragón-Mendizábal, Delgado-Casas, Navarro-Guzmán, MenachoJiménez et Romero-Oliva, 2016). Moins populaire, l'autre stratégie, qui consiste à prioriser la compréhension au détriment de la transcription, comporte elle aussi ses avantages et ses inconvénients. En effet, lorsque les apprenantes et les apprenants se concentrent sur la compréhension au détriment de la transcription, ils traitent les informations plus profondément, c'est-à-dire qu'ils les encodent dans leur mémoire à long terme (AragónMendizábal et al., 2016). Cependant, n'ayant pas constitué une mémoire 


\section{$\&$}

\section{REVUE HYBRIDE DE L'ÉDUCATION}

externe fiable par la PDN, ils s'exposent à l'oubli de $80 \%$ des informations entendues (Ozbay, 2005 cité dans Tahir, Tuncay, Ibrahim et Burak, 2013).

Quels gestes les enseignantes et les enseignants peuvent-ils poser afin d'amoindrir le risque de surcharges cognitives chez les apprenantes et les apprenants et ainsi leur permettre de se concentrer à la fois sur la compréhension et la transcription des informations ?

Les enseignantes et les enseignants ont un certain pouvoir sur la surcharge cognitive vécue par les apprenantes et les apprenants et peuvent poser certains gestes pour la diminuer.

D'abord, les enseignantes et les enseignants peuvent décider du format de la PDN. Par exemple, ils peuvent fournir un plan aux apprenantes et aux apprenants, dans lequel se trouve le squelette du discours ainsi que les idées principales avec de l'espace entre chacune pour prendre des notes, ce qui permet de réduire les risques de surcharge cognitive (Lorch, Lorch, et Matthews, 1985). En ayant en main le squelette du discours ou, en d'autres termes, la macrostructure du discours, l'apprenante ou l'apprenant n'a pas besoin de sélectionner les informations pertinentes et de les hiérarchiser, ce qui libère considérablement ses ressources attentionnelles, lui permettant de prendre des notes plus rapidement (Peverly et al., 2013). En plus, la littérature révèle que refléter la macrostructure d'un discours source dans les notes est un critère de qualité ou d'efficacité de la PDN (Williams et Eggert, 2002). Donc, le fait de fournir la macrostructure du discours source aux apprenantes et aux apprenants leur assure de prendre des notes minimalement complètes ou efficaces.

Dans la pratique, ces plans peuvent prendre diverses formes. Par exemple, dans la PDN en plan nommée « matrix note» (Kiewra et al., 1991), les idées et les sous-idées du cours (les idées essentielles) sont fournies dans un tableau par l'enseignante ou l'enseignant et ces dernières sont organisées dans l'espace de la page de façon à faire ressortir leur hiérarchisation ou les rapports qu'elles entretiennent entre elles : les idées principales se trouvent dans le haut de la page et les idées secondaires, dans la marge de gauche; le reste de l'espace permet à l'apprenante ou à l'apprenant de prendre des notes (figure 1). Donc, avec ce type de PDN, l'apprenante ou l'apprenant a, pour chaque page, une page qui traite d'une idée principale, laquelle est décortiquée en sous-idées. Avec ce type de plan, l'apprenante ou l'apprenant voit ses chances de surcharge cognitive diminuer, mais doit tout de même comprendre les informations pour sélectionner les plus pertinentes à noter, car la macrostructure du cours ne lui est pas entièrement fournie, ce qui permet lui de s'approprier les informations et de commencer à les mémoriser en les notant. 


\section{$\&$}

REVUE HYBRIDE DE L'ÉDUCATION

\begin{tabular}{|l|l|}
\hline \multicolumn{2}{|c|}{ Idée principal 1 } \\
\hline $\begin{array}{l}\text { Idée } \\
\text { secondaire 1 }\end{array}$ & \\
& \\
\hline $\begin{array}{l}\text { Idée } \\
\text { secondaire 2 }\end{array}$ & \\
\hline $\begin{array}{l}\text { Idée } \\
\text { secondaire 3 }\end{array}$ & \\
\hline
\end{tabular}

Figure 1 : Exemple de PDN en plan nommée « matrix note »

II est aussi possible pour l'enseignante ou l'enseignant de fournir un plan dans lequel la macrostructure du cours est encore plus précise, c'està-dire un plan dans lequel se retrouvent plus que les idées principales et secondaires (des idées des troisième et de quatrième niveaux) et dans lequel la hiérarchisation des idées est marquée explicitement par la numérotation (figure 2). Par contre, avec un tel plan, les apprenantes et les apprenants ont moins besoin de comprendre et de sélectionner les informations à noter puisque la macrostructure leur est presque entièrement fournie, ce qui n'encourage pas à être actifs lors de la PDN, ni de s'approprier ou de mémoriser les informations. En outre, fournir un plan aussi précis et complet soulève une question importante : comment les apprenantes et les apprenants peuvent-ils développer leur capacité à prendre des notes si la macrostructure (ou les notes) leur est presque entièrement fournie ? 


\section{$\&$}

REVUE HYBRIDE DE L'ÉDUCATION

\section{Idée principale 1}

\section{a. Idée secondaire 1}

\section{i. Sous-idée 1}

ii. Sous-idée 2

b. Idée secondaire 2

c. Idée secondaire 3

\section{i. Sous-idée 1}

\section{ii. Sous-idée 2}

\section{Idée principale 2}

\section{a. Etc.}

\section{Figure 2 : Exemple de PDN en plan avec macrostructure précise}

Par ailleurs, les gestes posés par l'enseignante ou l'enseignant, de façon planifiée ou non, pendant le cours peuvent déclencher ou inhiber la PDN et aider les apprenantes et les apprenants à sélectionner les informations pertinentes. L'enseignante ou l'enseignant peut opter pour les marqueurs de scripturalité ou les marqueurs d'oralité. Dans le cas des marqueurs de scripturalité, ce sont des gestes qui indiquent aux apprenantes et aux apprenants que l'information transmise oralement doit être écrite. Parmi ceux-ci se trouvent la notation au tableau ou encore, certains marqueurs prosodiques (longues pauses, rythme ralenti ou entrecoupé par des pauses, projection de la voix et absence d'hésitations) (Boch, 2000). Dans la pratique, les enseignantes et les enseignants peuvent user de ces marqueurs pour guider la PDN des apprenantes et des apprenants ou pour s'assurer qu'ils prennent bien en note toutes les informations essentielles du cours. Par exemple, pour guider la PDN des apprenantes et des apprenants et s'assurer qu'ils ne se perdent pas dans le fil de sa parole, l'enseignante ou l'enseignant pourrait inscrire au tableau les thèmes abordés sous la forme d'un plan au fur et à mesure. 


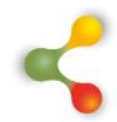

\section{REVUE HYBRIDE DE L'ÉDUCATION}

L'enseignante ou l'enseignant pourrait aussi noter ce plan au tableau dès le début du cours. De plus, afin de s'assurer que les apprenantes et les apprenants prennent bien les informations essentielles en note, l'enseignante ou l'enseignant peut mentionner ces dernières à la manière d'une dictée et les répéter plusieurs fois. L'enseignante ou l'enseignant peut aussi mentionner que ce qui est dit est important ou encore, demander explicitement aux apprenantes et aux apprenants de prendre certains éléments en note.

Dans le cas des marqueurs d'oralité, ce sont des gestes non planifiés et posés par l'enseignante ou l'enseignant afin de spécifier certains éléments de contenu. Ces gestes, n'étant pas planifiés par l'enseignante ou l'enseignant, peuvent être perçus comme des digressions au discours ou comme des fractures, et donc, perçus comme des informations qui ne sont pas importantes pour le contenu théorique pris en notes par les apprenantes ou les apprenants. Parmi ces marqueurs d'oralité qui inhibent la PDN se retrouvent les interactions entre les différentes actrices et les différents acteurs d'une classe, les questions, les réponses, les exemples spontanés donnés par l'enseignante ou l'enseignant, etc. (Boch, 2000). Par exemple, si une apprenante ou un apprenant pose une question par rapport à la théorie, qui a été ancrée dans la scripturalité parce que l'enseignante ou l'enseignant l'a planifiée dans ses notes et l'a donc inscrite au tableau ou a demandé aux apprenantes ou aux apprenants de la noter, la réponse que donnera l'enseignante ou l'enseignant sera plutôt ancrée dans l'oralité, car elle sera perçue comme une digression au discours théorique planifié. C'est comme si l'apprenante ou l'apprenant se disait que la réponse de l'enseignante ou de l'enseignant à la question n'étant pas inscrite dans les notes ou dans le plan de l'enseignante ou de l'enseignant, elle ne fait pas partie de la théorie; qu'elle n'est pas ancrée dans la scripturalité. Pourtant, la réponse fournie par l'enseignante ou l'enseignant peut être primordiale à la compréhension de l'élément théorique et peut parfois même avoir trait à un élément que l'enseignante ou l'enseignant a oublié de mentionner. II est donc important que l'enseignante ou l'enseignant prenne conscience qu'une réponse à une question (marqueur d'oralité) peut inhiber la PDN pour qu'il puisse contrer cet effet inhibitoire en usant de marqueurs de scripturalité qui déclenchent la PDN dans le cas où l'information de la question serait essentielle.

De plus, dans les cours, les échanges entre les pairs et entre les apprenantes et apprenants et l'enseignante ou l'enseignant en contexte de réalisation d'ateliers, par exemple, sont aussi ancrés dans l'oralité. II est alors important que l'enseignante ou l'enseignant situe consciemment les informations dans la scripturalité pour contrer l'effet inhibitoire des marqueurs d'oralité sur la PDN. Pour ce faire, l'enseignante ou l'enseignant peut, par exemple, noter les points essentiels qui ressortent des échanges au tableau, répéter certains éléments à la manière d'une dictée, demander directement de noter certains éléments, avertir les apprenantes et les apprenants dès le début des échanges qu'ils doivent prendre des notes, 


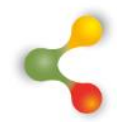

\section{REVUE HYBRIDE DE L'ÉDUCATION}

faire ressortir les points importants des échanges avec les apprenantes et les apprenants et les noter au tableau, laisser quelques minutes aux apprenantes et aux apprenants après les échanges et leur demander de faire une synthèse de ce qui a été dit, etc.

Bref, en étant conscient de l'effet des marqueurs de scripturalité et d'oralité sur la PDN des apprenantes et des apprenants, l'enseignante ou l'enseignant peut poser des gestes pour favoriser la PDN des éléments importants et permettre aux apprenantes et aux apprenants de concentrer leurs ressources attentionnelles sur d'autres opérations que la sélection des informations essentielles.

\section{Apports et prospectives}

En conclusion, la PDN est une activité complexe réalisée dans l'urgence qui donne bien du fil à retordre aux apprenantes et aux apprenants notamment en raison de la surcharge cognitive qu'elle peut occasionner. Pour éviter cette surcharge, les apprenantes et les apprenants usent stratégiquement de leurs ressources attentionnelles, mais cela ne leur permet pas nécessairement de maîtriser pleinement la PDN parce qu'ils se concentrent soit sur la compréhension, soit sur la transcription. Les enseignantes et les enseignants peuvent aussi aider à amoindrir les surcharges cognitives vécues chez les apprenantes et les apprenants en posant certains gestes, notamment en présentant la macrostructure du cours dans un document de notes, laquelle guide la sélection des informations importantes, ce qui permet aux apprenantes et aux apprenants d'user pleinement de la PDN et d'en arriver à mieux la maîtriser. D'autres auteures et auteurs, comme Romainville et Noël (2003), énoncent aussi le fait qu'une didactique de la PDN axée sur la métacognition devrait être mise en place dès le secondaire. Par les phases de modelage, d'entrainement et de rétroactions, l'enseignante ou l'enseignant pourrait amener les apprenantes et les apprenants à maîtriser la PDN. Par exemple, en réalisant des activités d'entrainement les apprenantes et les apprenants pourraient développer des microcompétences méthodologiques liées à la PDN, comme la capacité à abréger les mots, à structurer les notes, à reformuler, à résumer les informations entendues, à utiliser ultérieurement les notes, etc. Tout cela serait réalisé dans une perspective métacognitive où l'apprenante ou l'apprenant doit répondre à un questionnaire métacognitif afin de prendre conscience notamment des stratégies employées, des résultats qu'apportent ces stratégies, des éléments à améliorer et de la fonction de la PDN dans le but d'améliorer sa pratique. 


\section{REVUE HYBRIDE DE L'ÉDUCATION}

\section{Références}

Aragón-Mendizábal, E., Delgado-Casas, C., Navarro-Guzmán, J.-I., Menacho-Jiménez, I. et Romero-Oliva, M.-F. (2016). A Comparative Study of Handwriting and Computer Typing in Notetaking by University Students. Análisis comparativo entre escritura manual y electrónica en la toma de apuntes de estudiantes universitarios, 24(48), 101-107. doi: 10.3916/C48-2016-10

Boch, F. (1998). Pratiques d'écriture et de réécriture à l'université : La prise de notes, entre texte source et texte cible [thèse de doctorat inédite]. Université Stendhal.

Boch, F. (2000). Prise de notes et écriture conceptuelle à l'université. Pratiques, 105-106, 137-158.

Boyle, J. R. et Forchelli, G. A. (2014). Differences in the note-taking skills of students with high achievement, average achievement, and learning disabilities. Learning and Individual Differences, 35, 9-14. doi: 10.1016/j.lindif.2014.06.002

Branca-Rosoff, S. (1998). Abréviations et icônes dans les prises de notes des étudiants. Dans M. Bilger, K. v. d. Eynde, et F. Gadet (dir.), Analyse linguistique et approches de l'oral : recueil d'études offert en hommage à Claire Blanche-Benveniste (p. 385). Peeters.

Cary, M. et Carlson, R. A. (2001). Distributing Working Memory Resources During Problem Solving. Journal of Experimental Psychology: Learning, Memory, and Cognition, 27(3), 836-848. doi: 10.1037/0278-7393.27.3.836

Hartley, J. (2002). Notetaking in non-academic settings: a review. Applied Cognitive Psychology, 16(5), 559-574. doi: 10.1002/acp.814

Kiewra, K. A., Dubois, N. F., Christian, D., McShane, A., Meyerhoffer, M. et Roskelley, D. (1991). Note-Taking Functions and Techniques. Journal of Educational Psychology, 83(2), 240-245. doi: 10.1037/0022-0663.83.2.240 


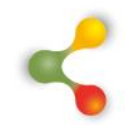

\section{REVUE HYBRIDE DE L'ÉDUCATION}

Lorch, R. F., Lorch, E. P. et Matthews, P. D. (1985). On-line processing of the topic structure of a text. Journal of Memory and Language, 24(3), 350-362. doi: 10.1016/0749-596X(85)90033-6

Makany, T., Kemp, J. et Dror, I. E. (2009). Optimising the use of note-taking as an external cognitive aid for increasing learning. British Journal of Educational Technology, 40(4), 619-635. doi: 10.1111/j.14678535.2008.00906.x

Paivandi, S. (2015). Apprendre à l'université. De Boeck Supérieur.

Peverly, S. T. et Sumowski, J. F. (2012). What Variables Predict Quality of Text Notes and are Text Notes Related to Performance on Different Types of Tests?. Applied Cognitive Psychology, 26(1), 104-117. doi: 10.1002/acp.1802

Peverly, S. T., Vekaria, P. C., Reddington, L. A., Sumowski, J. F., Johnson, K. R. et Ramsay, C. M. (2013). The Relationship of Handwriting Speed, Working Memory, Language Comprehension and Outlines to Lecture Note-taking and Test-taking among College Students. Applied Cognitive Psychology, 27(1), 115-126. doi: 10.1002/acp.2881

Piolat, A. (2004). Approche cognitive de l'activité rédactionnelle et de son acquisition. Le rôle de la mémoire de travail. Linx, 51, 55-74.

Piolat, A. (2010). Approche cognitive de la prise de notes comme écriture de l'urgence et de la mémoire externe. Le français d'aujourd'hui, 170(3), 51-62.

Piolat, A. et Boch, F. (2004). Apprendre en notant et apprendre à noter. Dans E. Gentaz et P. Dessus (dir.), Comprendre les apprentissages. Psychologie cognitive et éducation ( $\mathrm{p}$ 133-152). Dunod. http://lidilem.u-grenoble3.fr/IMG/pdf/piolatboch.pdf

Pollet, M.-C. (2001). Pour une didactique des discours universitaires. Étudiants et système de communication à l'université. De Boeck Supérieur. 


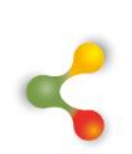

\section{REVUE HYBRIDE DE L’ÉDUCATION}

Romainville, M. et Noël, B. (2003). Métacognition et apprentissage de la prise de notes à l'université. Arob@se, (1-2), 87-96.

Roussey, J.-Y. et Piolat, A. (2003). Prendre des notes et apprendre. Effet du mode d'accès à l'information et de la méthode de prise de notes. Arob@se, (1-2), 47-68.

Tahir, G., Tuncay, D., Ibrahim, C. et Burak, D. (2013). The impact of notetaking while listening on listening comprehension in a higher education context. International Journal of Academic Research Part B, 5(1), 93-97. doi: 10.7813/2075-4124.2013/5-1/B.16

Williams, R. L. et Eggert, A. C. (2002). Notetaking in college classes: student patterns and instructional strategies. The Journal of General Education, 51(3), 173-199. doi: 10.1353/jge.2003.0006 